\title{
MAORY design trade-off study: tomography dimensioning
}

\author{
Sylvain Oberti ${ }^{\mathrm{a}}$, Miska Le Louarn ${ }^{\mathrm{a},}$ Emiliano Diolaiti $^{\mathrm{b}}$, Carmelo Arcidiacono $^{\mathrm{b}}$, Laura Schreiber ${ }^{\mathrm{b}}$, \\ Matteo Lombini ${ }^{\mathrm{b}}$, Fausto Cortecchia ${ }^{\mathrm{b}}$ \\ ${ }^{a}$ European Southern Observatory, Karl Schwarzschildstr. 2, 85748 \\ Garching bei Muenchen, Germany \\ ${ }^{\mathrm{b}}$ INAF - Osservatorio Astronomico di Bologna, Via P. Gobetti 93/3, 40129 \\ Bologna, Italy
}

\begin{abstract}
MAORY is the MCAO module that will equip the Nasmyth A of the E-ELT and feed the instrument MICADO as well as a potential second port instrument. In this paper, by means of end-to-end simulations, we will study the main dimensioning parameters that impact the tomography performance. The explored parameter space will cover the number of post-focal Deformable Mirrors, their pitches and conjugation altitude as well as the number of Laser Guide Stars and their position on the sky. The simulation results will support the design trade-off aiming at maximizing the Adaptive Optics performance in a field of view of up to $3^{\prime}$ in terms of strehl ratio, uniformity and NGS sky coverage. Sensitivity studies will highlight the robustness of these design choices to the varying conditions of observation such as seeing, turbulence profile and zenith distance.
\end{abstract}

Keywords: Multi-conjugate Adaptive Optics RelaY (MAORY), Multi-Conjugate Adaptive Optics (MCAO), Deformable Mirror (DM), Wave Front Sensor (WFS), Laser Guide Star (LGS), Natural Guide Star (NGS)

\section{INTRODUCTION}

In the framework of MAORY phase B ([1], [2], [3]), the main design trade-offs originating from phase A are revisited. In particular, the high-level parameters influencing the hardware design shall be frozen as early as possible before PDR, e.g. the number, conjugation altitude and pitch of the post-focal DMs or the number of LGS and their geometry. This paper presents the analyses of such major design trade-off supported by the end-to-end simulation tool called OCTOPUS, used at ESO to simulate the AO systems for the E-ELT ([4]). The MCAO tomography is based on the FRIM3D algorithm ([5]) implemented into OCTOPUS. This work is complementary to the other end-to-end simulations performed with simulation tool ([6],[7]) developed by C. Arcidiacono at INAF Bologna. However, necessary overlaps allow cross validating the two codes and consolidating the results.

\section{SCOPE}

The main objective of this study is to investigate the dimensioning parameters constrained by tomographic and generalized fitting error

- $\quad$ Number of post-focal DMs conjugated in altitude

- DM pitch

- $\quad$ DM altitudes

- LGS asterism angle

- $\quad$ Number of LGS

- $\quad$ LGS and NGS asterism geometry

under some environmental constraints such as seeing, $\mathrm{Cn} 2$ profile, telescope elevation angle, number of LGSs or M1 central obstruction 


\section{END-TO-END SIMULATION TOOL}

The analyses presented in this paper have been performed with Octopus, ESO's end-to-end simulation tool. The tomography reconstruction is based on FRIM3D which is a fractal iterative reconstruction algorithm including MMSE regularization and open loop priors (POLC: Pseudo-Open Loop Control). The DM command computation is performed in two steps:

- Tomographic reconstruction to estimate the multi-layer turbulent phase: FRIM3D/POLC with perfect priors on $\mathrm{Cn}^{2}$ profile

- Projection of the estimated 3D phase onto the DMs space. The projection is optimized over a specific FoV, defined by the lines of sight represented by the squares on Figure 1

The simulations presented in this paper emulate some major terms of the error budget: the tomographic error, the generalized fitting error, the spatial aliasing and the temporal error. However, it was decided to neglect WFSs' noise (high flux and no LGS elongation) since we focus here on deterministic geometry trade-off and sensitivity to environment.

The telescope is simulated with an M1 diameter of $37 \mathrm{~m}$ sampled on a support of $740 \times 740$ pixels (1200x 1200 for the highest meta-pupil) and with a central obstruction of $28 \%$ unless specified otherwise. At a frame rate of $500 \mathrm{~Hz}(2$ frames delay), MAORY controls M4 (square geometry, pitch $0.5 \mathrm{~m}$, bilinear influence function) conjugated at $612 \mathrm{~m}$ and one or two post focal DM(s) with a similar model but conjugated at higher altitude(s) and with coarser pitch(es). The Wavefront is analyzed by 6 LGS WFS with $74 \times 74$ subapertures with $6 \times 6$ pixels in each. A FoV of 2.4 " is sufficient to emulate a fictive non-elongated LGS. There is no read-out noise and the flux is of 10000 photoelectrons per subaperture per frame. Finally, 3 noiseless fast NGS WFS measure in H band and reconstruct tomographically the Tip/Tilt, Defocus and Astigmatisms. No truth sensor is simulated here. The turbulence is simulated by a profile of 35 layers with an outer scale of $25 \mathrm{~m}$. Several Cn2 profiles have been analyzed in the framework of sensitivity studies. Some examples of Cn2 profiles are plotted in Figure 2. The loop performance is generally evaluated in K band (2.2 microns) on several stars paving the FoV (blue crosses in Figure 1)

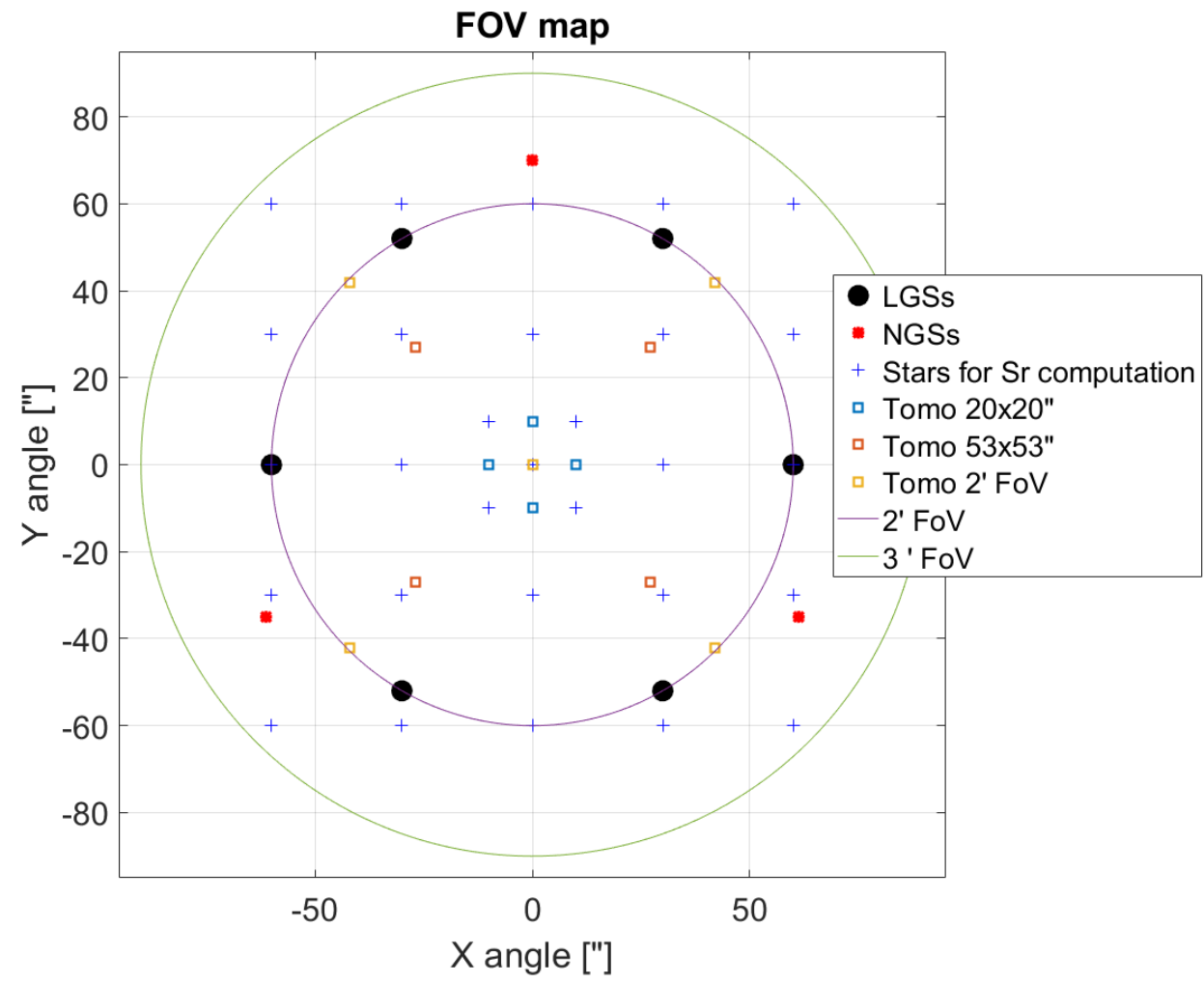

Figure 1: Example of FoV map - LGSs (black disks) at 1' radius - NGSs (red stars) at 70" radius - Stars for Sr computation (blue crosses) - Stars for iterative Sr optimization in the FoV (squares) 


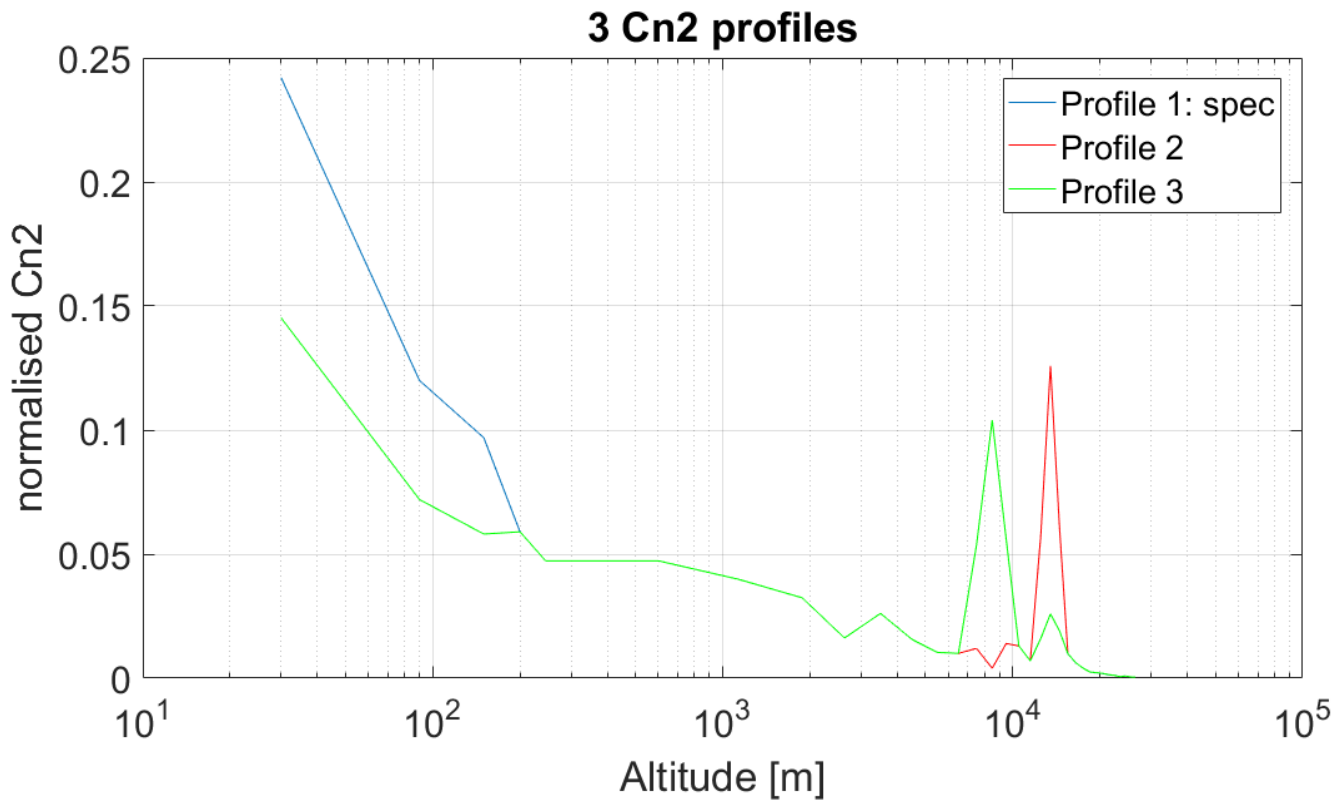

Figure 2: 3 normalized $\mathrm{Cn} 2$ profiles used as inputs for simulation. [Blue] Profile 1: median E-ELT specified profile with 75 $\%$ of the energy within the first 1000 meters [Red] Profile 2: transfer of a third of the ground energy into the jet stream layer so that 50\% remains within the first 1000 meters and an additional turbulence peak @ $13.5 \mathrm{~km}$ [Green] Profile 3 with peak (a) $8.5 \mathrm{~km}$ and $50 \%$ of the energy remaining within the first 1000 meters

\section{NUMBER OF POST-FOCAL DM AND PITCH}

In this section, we summarize the results of the analyses concerning the number of post-focal DMs and their pitch. Figure 3 and Figure 4 show the impact of DM pitch when two or one post-focal DMs are introduced into MAORY's design, for two significantly different turbulence profiles.

Figure 5 illustrate the additional robustness to $\mathrm{Cn} 2$ profile variation provided by a $2^{\text {nd }}$ post-focal DM in presence of an additional turbulence layer at an altitude far from the conjugation altitude of the DMs.

Based on these results and other studies reported in this paper, the conclusion is that two post-focal DMs are desirable in order to enhance the:

- Performance in the technical field: sky coverage and robustness (acquisition)

- Performance in the blue for NGS sky coverage and MICADO performance

- Robustness to $\mathrm{Cn} 2$ profile variation and zenith distance

- Performance in the science field for low theta0 turbulence cases 

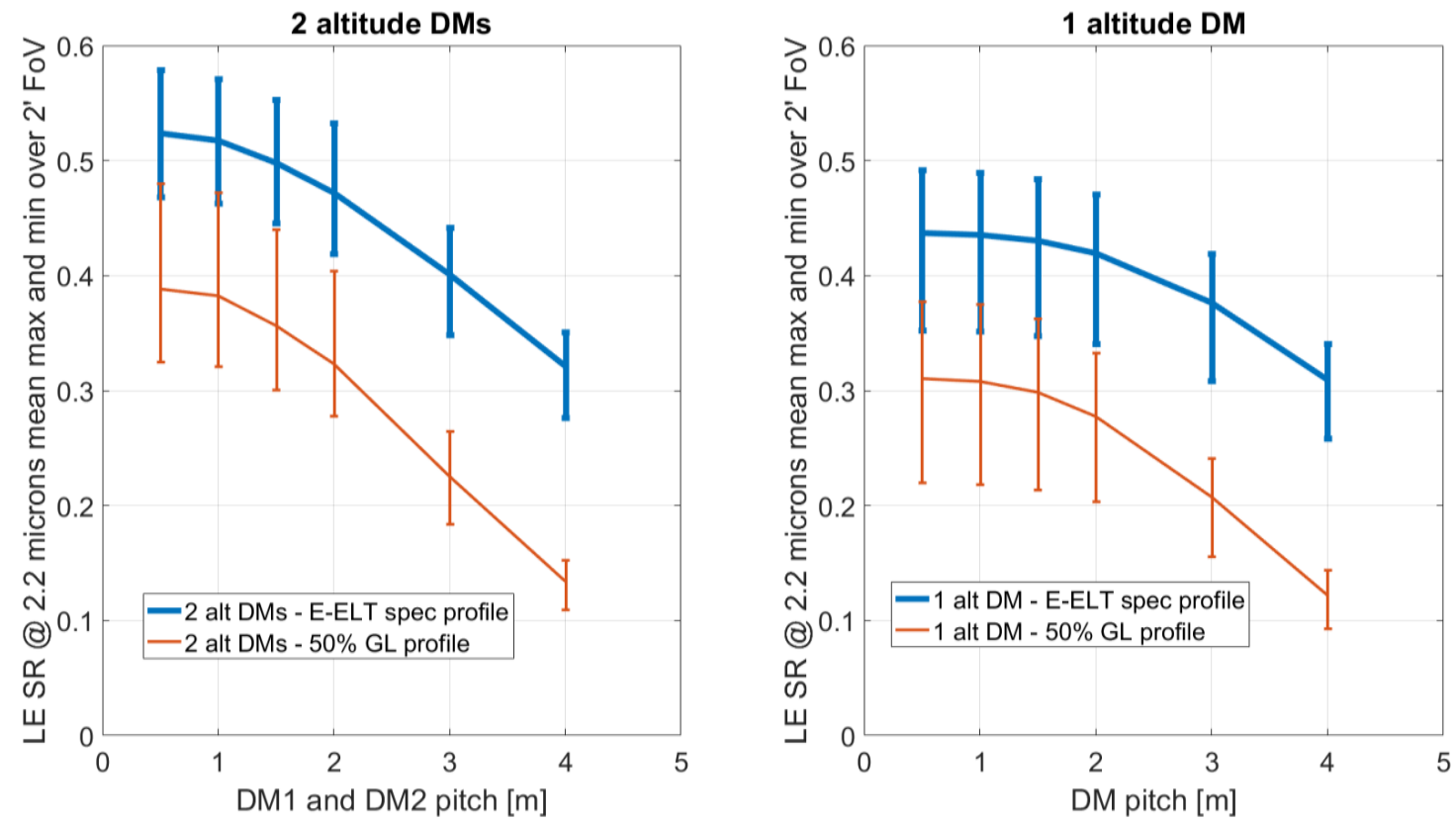

Figure 3: roo $=0.129 \mathrm{~m}, \mathrm{Z}=30^{\circ}$ - Mean, max and min SR across 2' GoV plotted against DM pitch [Left] 2 altitude DIs @ 4 $\mathrm{km}$ and $12.7 \mathrm{~km}$ with identical pitch for $2 \mathrm{Cn} 2$ profiles (Profile 1: blue, Profile 2: red) [Right] 1 altitude DM @12.7 km for 2 $\mathrm{Cn} 2$ profiles (Profile 1: blue, Profile 2: red)

Profile 1

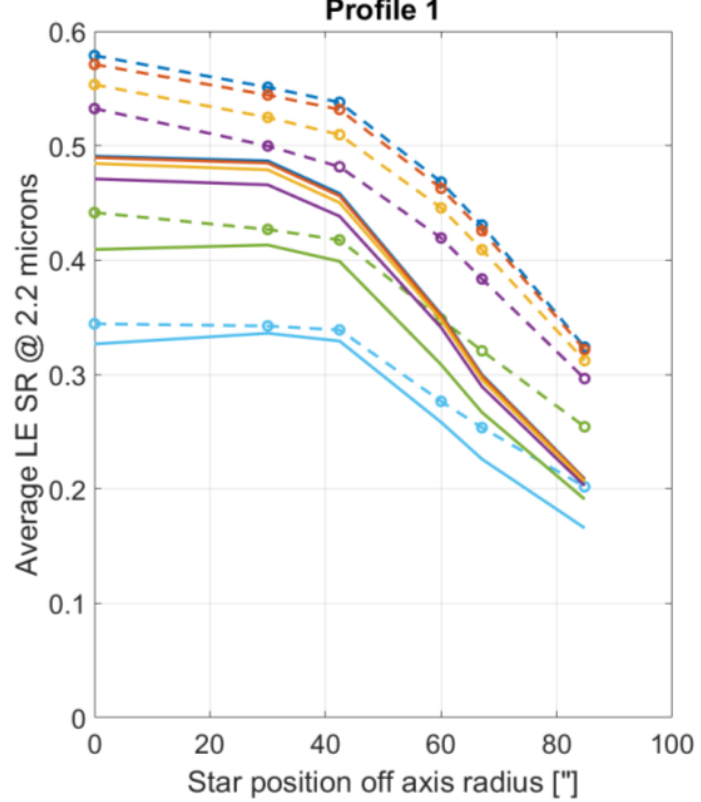

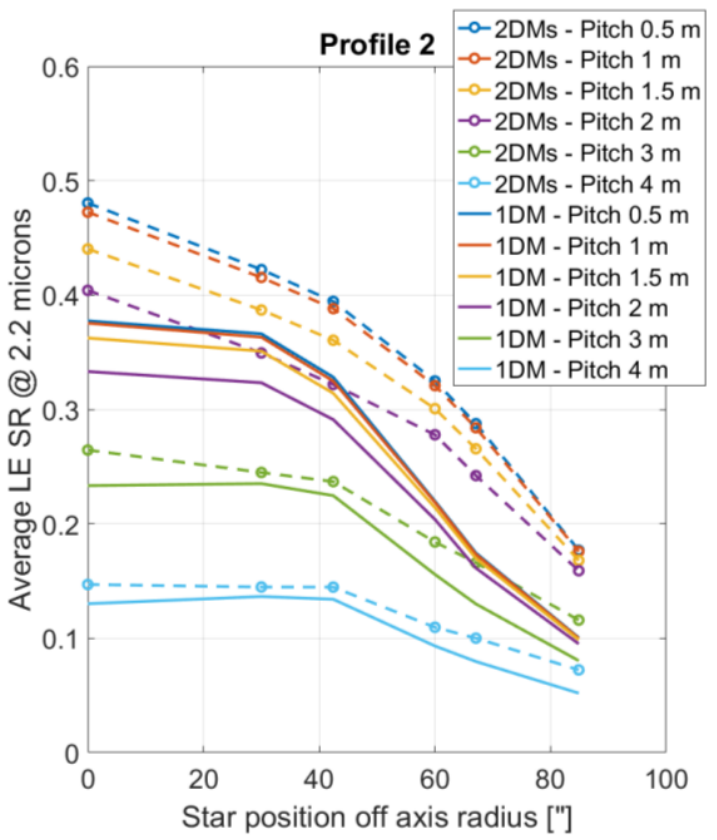

Star position off axis radius ["]

Figure 4: $\mathrm{r} 0=0.129 \mathrm{~m}, \mathrm{Z}=30^{\circ}$ - Average long exposure SR against star off axis radius for 2 altitude PMs (dashed lines) and 1 altitude DM (solid lines) for 5 different pitch values [Left] Profile 1 [Right] Profile 2 


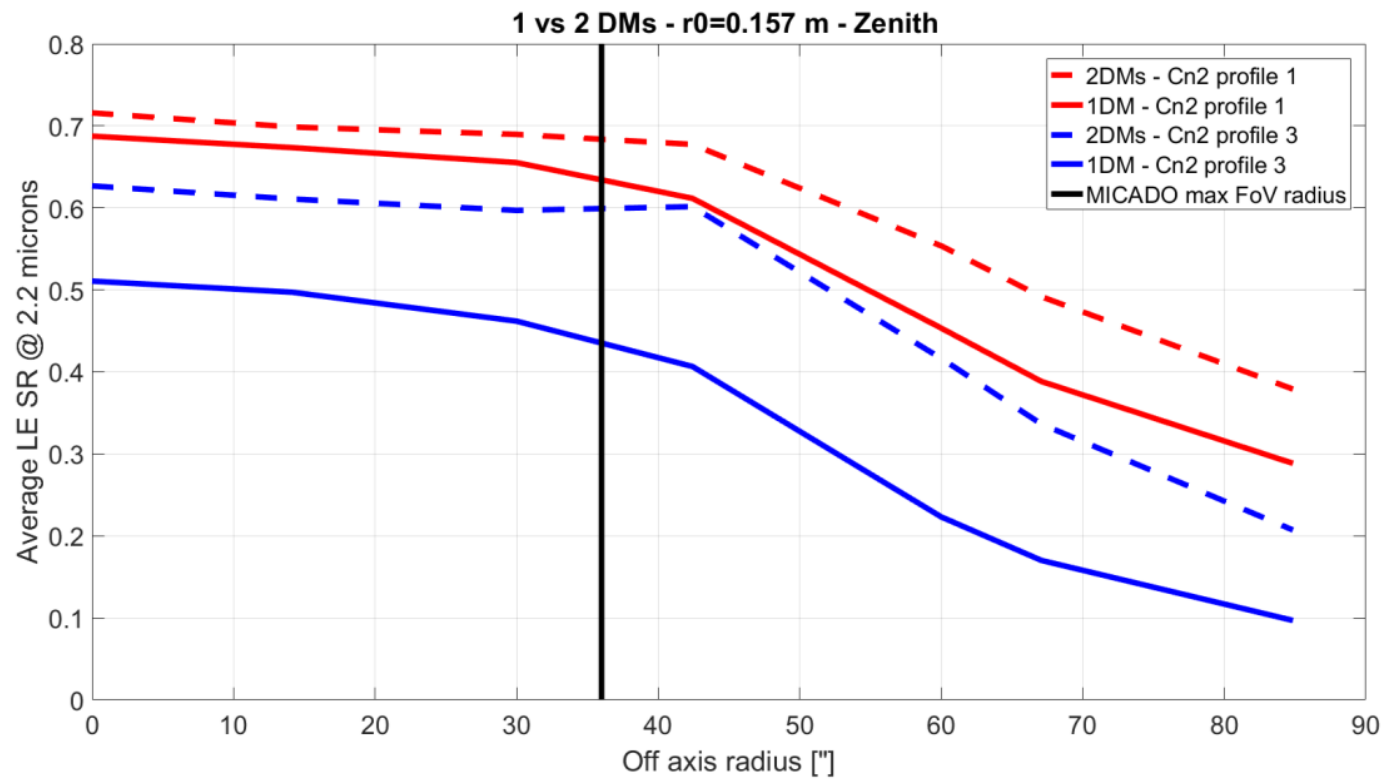

Figure 5: $\mathrm{r} 0=0.157 \mathrm{~m}, \mathrm{Z}=0^{\circ}$ - Illustration of the higher performance and better robustness to $\mathrm{Cn} 2$ profile variation provided by two post-focal DMs w.r.t. one post-focal DM - Radial SR for 2 Turbulence profiles - Profile 1 [red] and 3 [blue] - with 1 [solid line] or 2 [dashed line] post-focal DMs - Pitch 1.5 m - LGS @ 45" radius

\section{NUMBER OF LGSS AND M1 CENTRAL OBSTRUCTION}

This In this section, we investigate the impact of E-ELT phase 1 and phase 2 configurations, limiting our study to the two following features:

- M1 central hole: $28 \%$ for phase 2 and $57 \%$ for phase 1 (called doughnut hereafter)

- $\quad$ Number of LGSs: 6 for phase 1 and 4 for phase 2

Note that only tomographic effects are investigated here. The is an obvious impact of M1 with $57 \%$ central obstruction: the NGS sky coverage will be strongly reduced due to the smaller collecting area and the additional measurement noise brought by PSF elongation when truncating a $20 \mathrm{~m}$ subaperture. These effects are not studied here since we consider a bright NGS.

\subsection{K band performance}

In $\mathrm{K}$ band, the following trends can be highlighted:

- M1 doughnut impact on $\mathrm{Sr}$ is not significant, when assuming a bright NGS

- There is a significant impact on performance when going from 6 LGSs to 4 LGSs

- In the E-ELT phase 1 case, the tomographic error (due to fewer LGSs and a worse sampling of the meta-pupil due to a larger central obstruction of the pupil) dominates over the fitting error. In this case:

$\circ$ The pitch value does not have a significant impact in the range $1 \mathrm{~m}$ to $2 \mathrm{~m}$

○ It is hard to justify the need of two post-focal DMs

- The benefit when upgrading from 1 post-focal DM to 2 post-focal DMs increases with:

○ lower pitch

○ more LGSs

- E-ELT phase $1 \rightarrow$ phase 2 
- Towards the edge of the FoV (outside the scientific FoV), where the IR NGS are picked up. This way, the sky coverage would be increased.

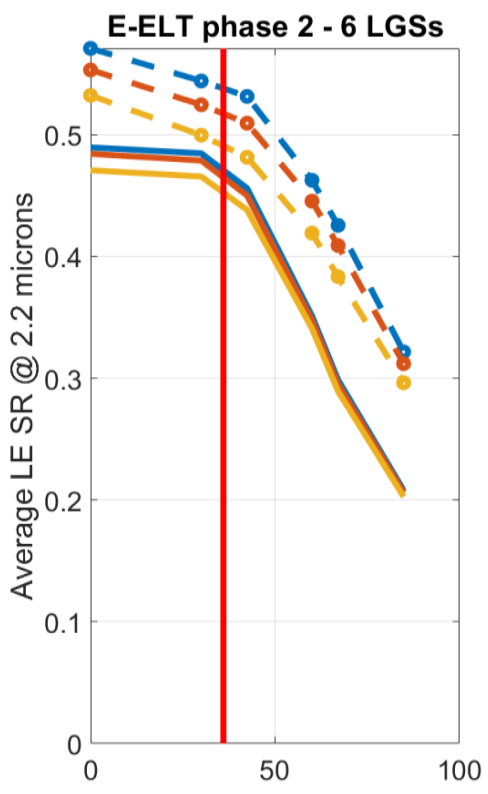

Star position off axis radius ["]

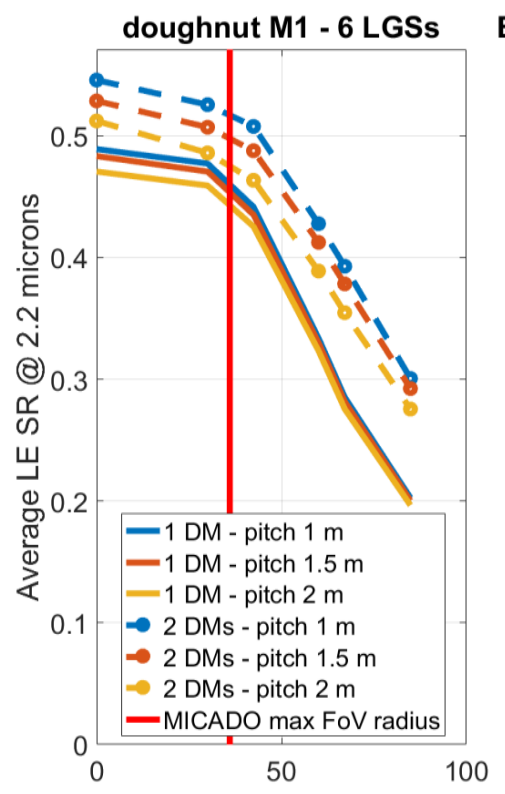

Star position off axis radius ["]

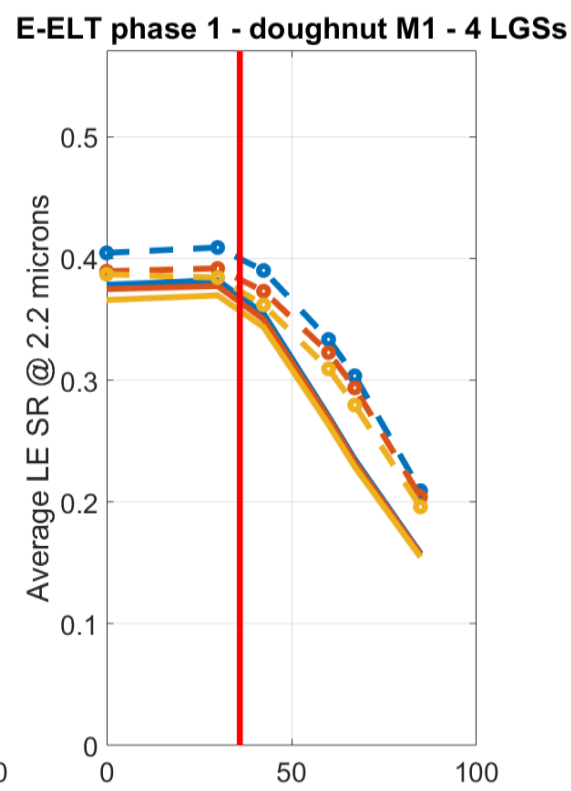

Star position off axis radius ["]

Figure 6: Average long exposure SR @ 2.2 microns against star off axis radius for 2 altitude DMs (dashed lines) and 1 altitude DM (solid lines) for 5 different pitch values [Left] E-ELT phase 2 [Right] E-ELT phase 1 [Middle] Hybrid case with doughnut M1 and 6 LGSs

\section{$5.2 \mathrm{~J}$ band performance}

It is worth noting that the $\mathrm{K}$ band technical specifications seem achievable in all cases studied in the previous subsection. Without accounting for several major terms of the error budget (low flux, elongation, telescope residual WFE, etc.), it seems that there is sufficient margin with respect to the specified performance in median conditions, i.e. a Sr of $0.3 @$ 2.2 microns within 1' diameter FoV.

Nevertheless, even if not specified, the performance at lower wavelengths is important for several MICADO science cases, especially stellar population studies. Therefore, to enhance the differences observed between the various trade-off options, the phase variance is computed from the previously simulated $\mathrm{K}$ band $\mathrm{Sr}$, scaled to $\mathrm{J}$ band to allow computing a coherent energy at 1.25 microns. The following figures exhibit a "magnification" of the various impacts:

- Going from 4 to 6 LGSs dramatically increases performance by factors between 2 and 3 depending on the number of DMs and pitch, allowing to reach diffraction limited PSFs cores in $\mathrm{J}$ band.

- With 6 LGSs, the upgrade from 1 to 2 post-focal DMs provides a performance gain of up to $50 \%$ within the MICADO FoV with the lowest pitch case.

- The performance gain with 2 post-focal DMs is much higher in the technical field, reaching scale factors higher than 3. It would strongly improve the NGS sky coverage in the blue end. This gain at the edge of the field could also be useful for the second port instrument.

- The impact of fitting error is more spectacular than in $\mathrm{K}$ band, which confirms once again that a smaller pitch is desirable, if 6 LGSs are available. This should be even truer when considering worse seeing cases of further zenith distance.

- Finally, these results confirm that 6 LGSs should have a high priority within the E-ELT project. As far as MAORY is concerned, the ratio performance gain vs. cost is indeed much higher than the one achieved by populating M1 segments. Performance should here be understood as Strehl ratio, not PSF shape or sky coverage that would suffer more from M1 central obstruction. 


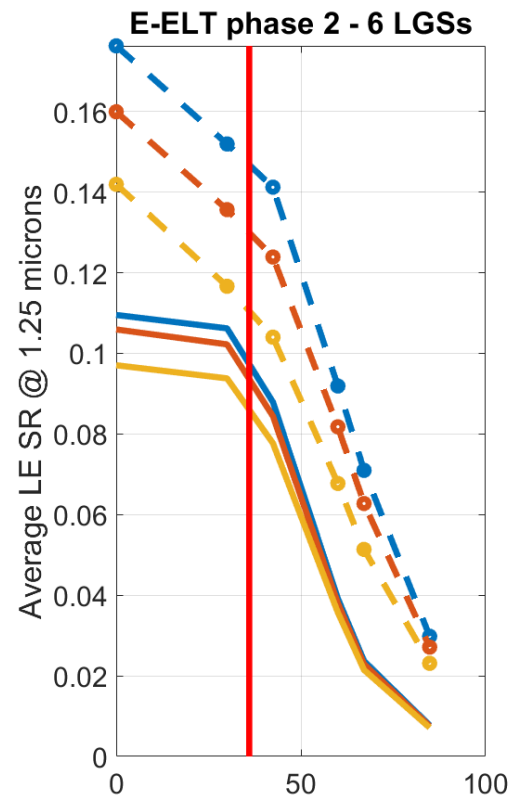

Star position off axis radius ["]

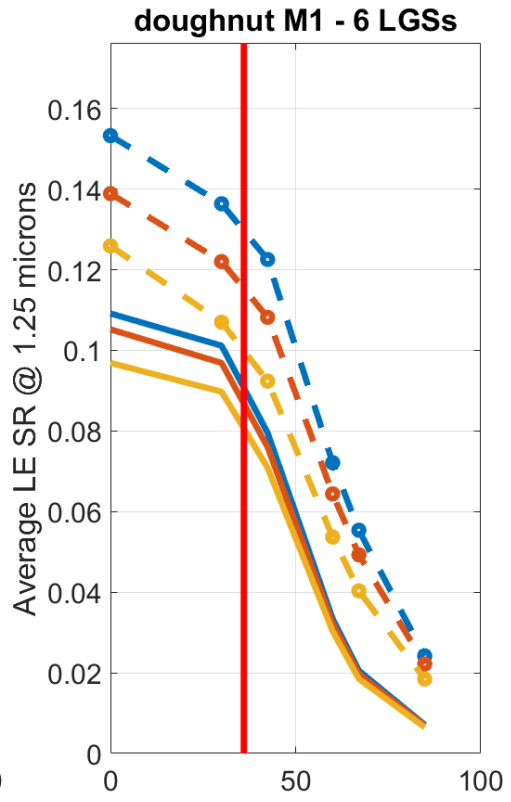

Star position off axis radius ["]
E-ELT phase 1 - doughnut M1 - 4 LGSs

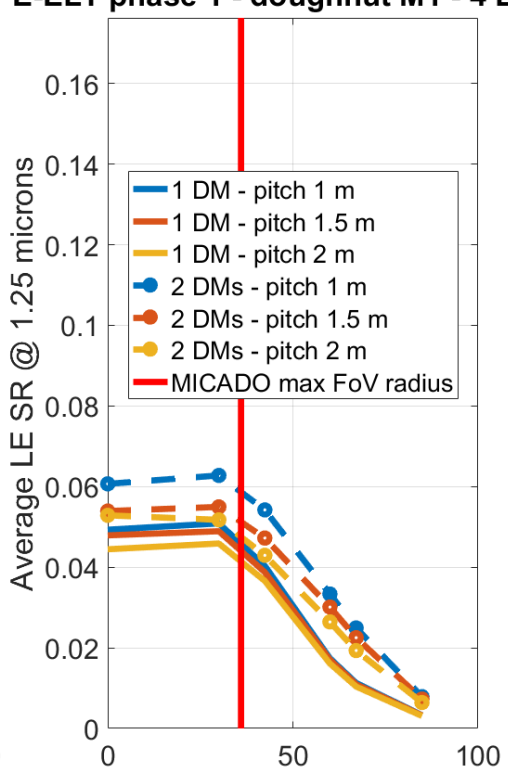

Star position off axis radius ["]

Figure 7: Average long exposure SR @ 1.25 microns against star off axis radius for 2 altitude DMs (dashed lines) and 1 altitude DM (solid lines) for 5 different pitch values [Left] E-ELT phase 2 [Right] E-ELT phase 1 [Middle] Hybrid case with doughnut M1 and 6 LGSs

\section{POST-FOCAL DM ALTITUDE}

The post-focal DM(s) will be located at a fixed position on the Nasmyth platform. Thus, it will not be possible to track the turbulence altitude by conjugating the DMs at variable altitude, in particular against telescope elevation. Therefore, the conjugation altitude of the post-focal $\mathrm{DM}(\mathrm{s})$ shall be determined as a trade-off across the operating range.

\subsection{Single post-focal DM case}

With profile 1, in the case of one altitude DM with a pitch of $1 \mathrm{~m}$, the DM altitude is scanned between 6 and $16 \mathrm{~km}$. The effect on performance is significant in this case. The phase A conjugation altitude of $12.7 \mathrm{~km}$ seems reasonable when considering the specified E-ELT Cn2 profile close to zenith. However, a higher conjugation altitude of about $16 \mathrm{~km}$ would provide more margin towards larger zenith distances.
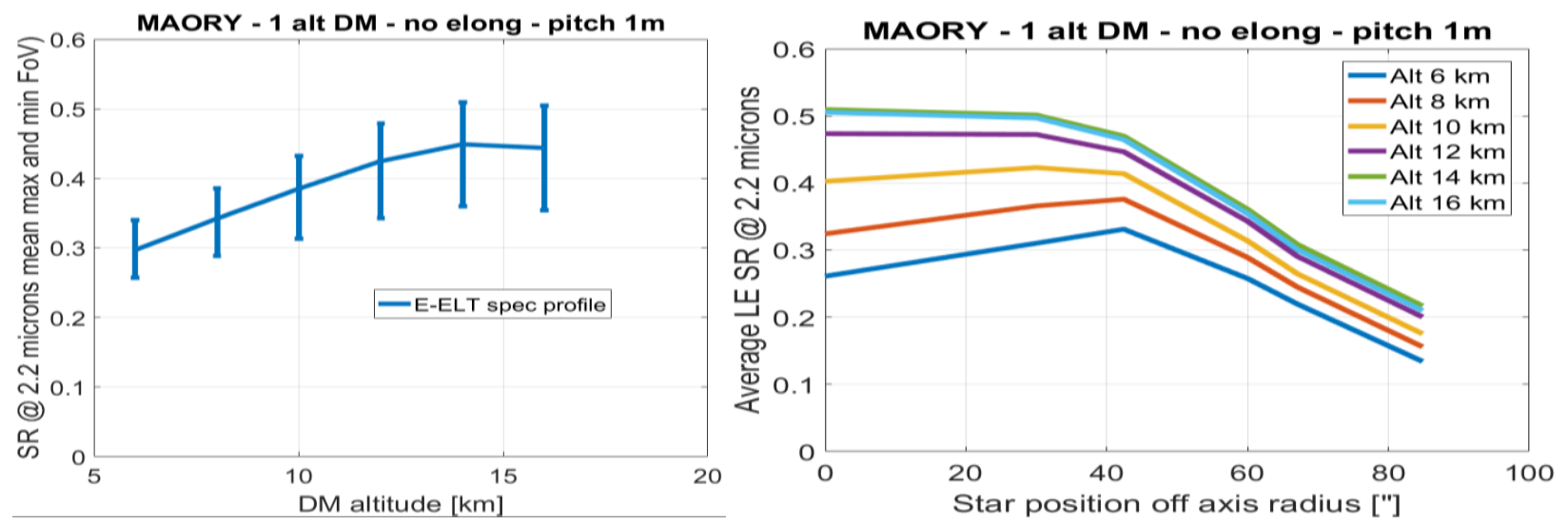

Figure 8: $\mathrm{r} 0=0.129 \mathrm{~m}, \mathrm{Z}=30^{\circ}$ - [Left] Mean, $\max$ and $\min \mathrm{SR}$ across ${ }^{\prime}$ FoV plotted against single DM altitude [Right] Average long exposure SR against star off axis radius for 1 altitude DM 


\subsection{Two post-focal DMs case}

With two post-focal DMs and a range of zenith distances $\left(0,45\right.$ and $\left.60^{\circ}\right)$, the following conclusions can be drawn:

- The altitude of the lower post-focal DM is not sensitive. It will be located where most convenient in terms of optical design

A $16 \mathrm{~km}$ conjugation altitude for the higher post-focal DM is a good trade-off across the range of zenith distances. This conjugation altitude of $16 \mathrm{~km}$ has been confirmed by a sensitivity study based on three $\mathrm{Cn} 2$ profiles extracted as the most representative case of a stereo-scidar measurement campaign at Paranal. Furthermore, this altitude has the advantage to be optimal whether one or two post-focal DM(s) are baselined.
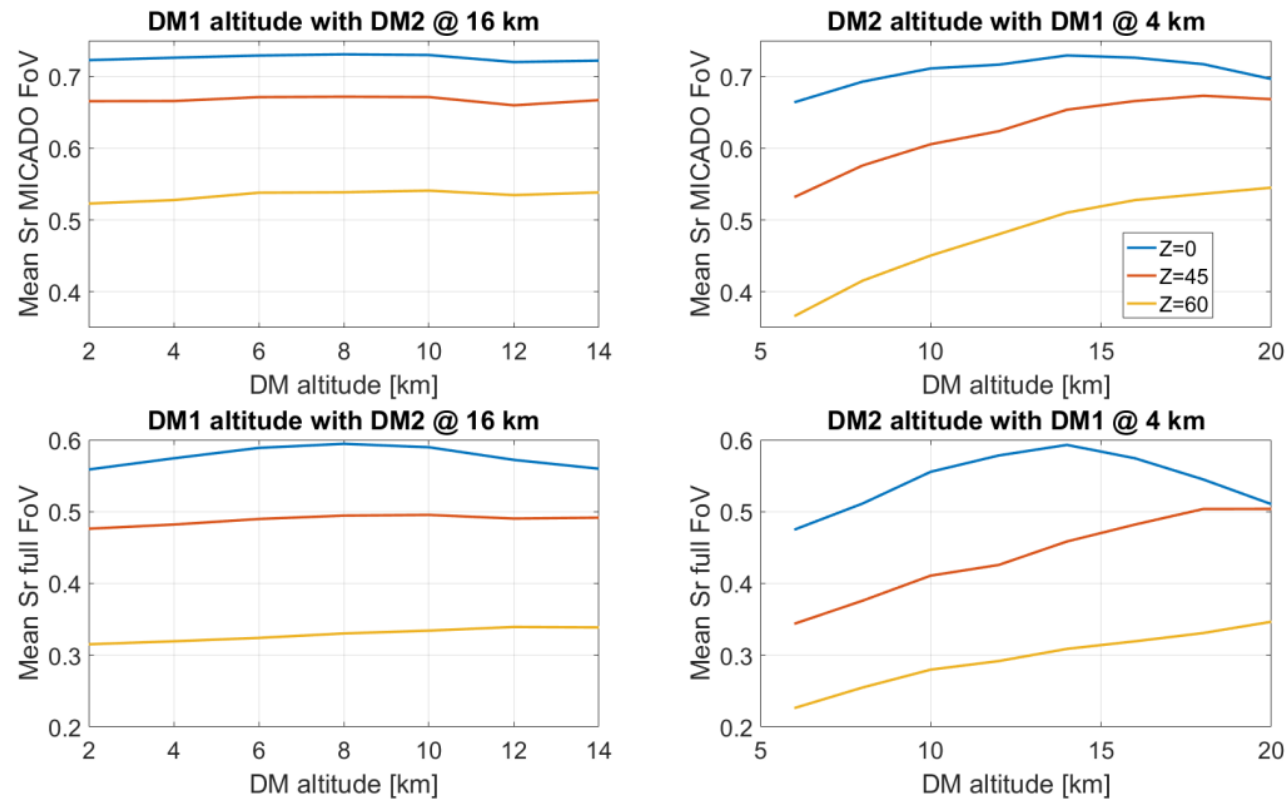

Figure 9: Performance against post-focal DM altitude [Left] DM2@16 km and DM1 spanning 2 to $14 \mathrm{~km}$ altitude [Right] DM1 @ 4 km and DM2 spanning 6 to $20 \mathrm{~km}$ altitude [Top] Mean SR (2.2 microns) in the MICADO large field [Bottom] Mean SR (2.2 microns) in the full field for 3 zenith distances: $0^{\circ}$ [blue], $45^{\circ}$ [red] and $60^{\circ}$ [yellow] - r0 $=0.157 \mathrm{~m}$

\section{LGS ASTERISM ANGLE}

In this section, we analyze the optimal angle of the LGS constellation.

\subsection{Full field optimization}

When optimizing the tomography across the full field of 2 arcminutes diameter, the average strehl ratio across the field depends on the LGS constellation angle as shown on Figure 10. Naturally, the performance towards the center of the FoV increases with smaller LGS constellations while the uniformity of the performance across the field increases with larger LGS constellations. The best trade-off between the scientific field (MICADO large field is 72" diameter) and the technical field (NGS sky coverage) is obtained for LGS constellation radii between 45 and 60". 


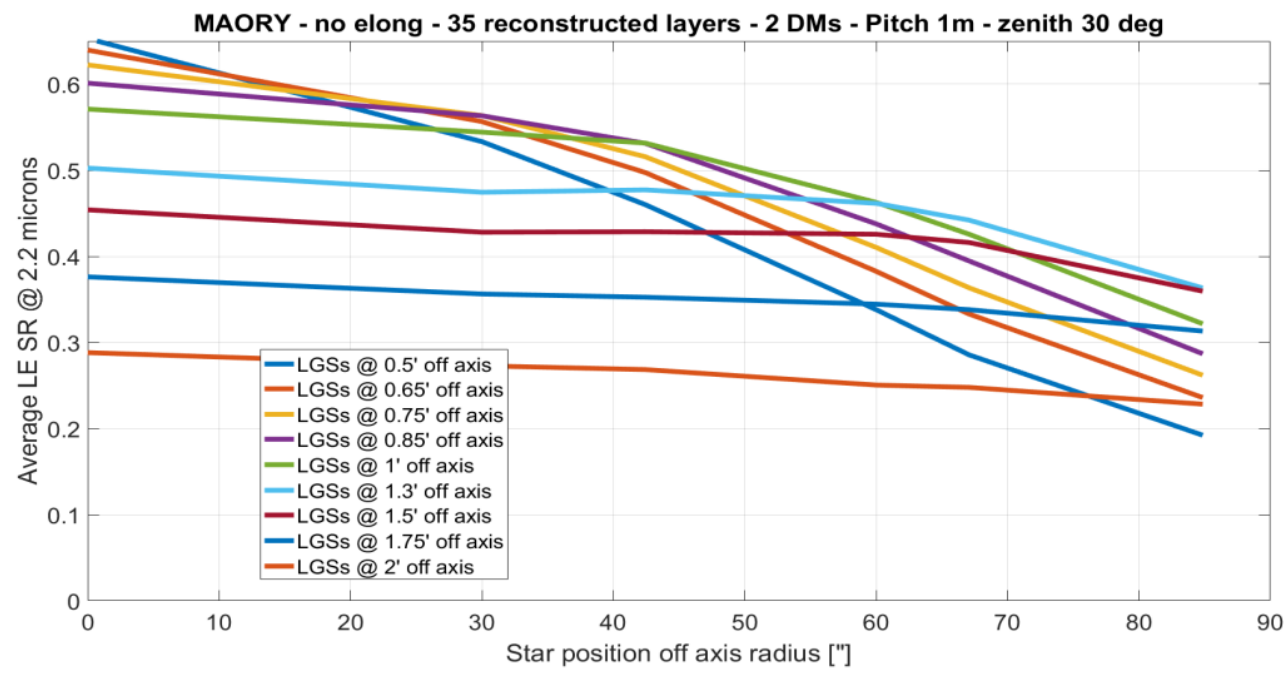

Figure 10: $\mathrm{r} 0=0.129 \mathrm{~m}, \mathrm{Z}=30^{\circ}$, pitch $1 \mathrm{~m}$ - Average long exposure SR against star off axis radius for different LGS asterism angles

\subsection{Optimized tomography}

In the previous section, the MCAO correction was optimized over the full field of view. However, it is possible to project the reconstructed phase along different lines of sight to privilege a particular area of the FoV. In order to evaluate the optimal LGS constellation angle, we investigate three projection optimization FoVs in K band with one star at the center of the FoV and:

- FoV1: 4 stars on the sides of a square of 20" side

- FoV2: 4 stars at the corners of a square of 53" side

- FoV3: 4 stars at the corners of a square of 2' diagonal

The results are shown in Figure 11 and allow drawing the following conclusions:

- The maximum and average Strehl ratio across the MICADO field is highest for LGS @ 45" radius

- The average performance in 2' FoV is highest for LGS @ 1' off axis

- At zenith, the performance provided by the optimization across the smallest field (FoV1) is not better than the one obtained with FoV2 or FoV3. This is because the NGSs are far off axis and the Sr optimized at the center of the field, hence the NGS PSFs are badly corrected. In addition, the NGS WFS FoV had not been set to a sufficiently large value to cope with such bad PSF.

In the next section, the NGS FoV WFS is then increased and the NGSs positioned closer to the axis, in order to investigate the "ultimate performance" configuration, with an airmass set to 2 (zenith distance $=60^{\circ}$ ). This extreme case is selected on purpose to drive the optimal LGS constellation angle towards narrow asterism. Our goal is to verify is a narrow asterism could be pertinent for MICADO's small field. 

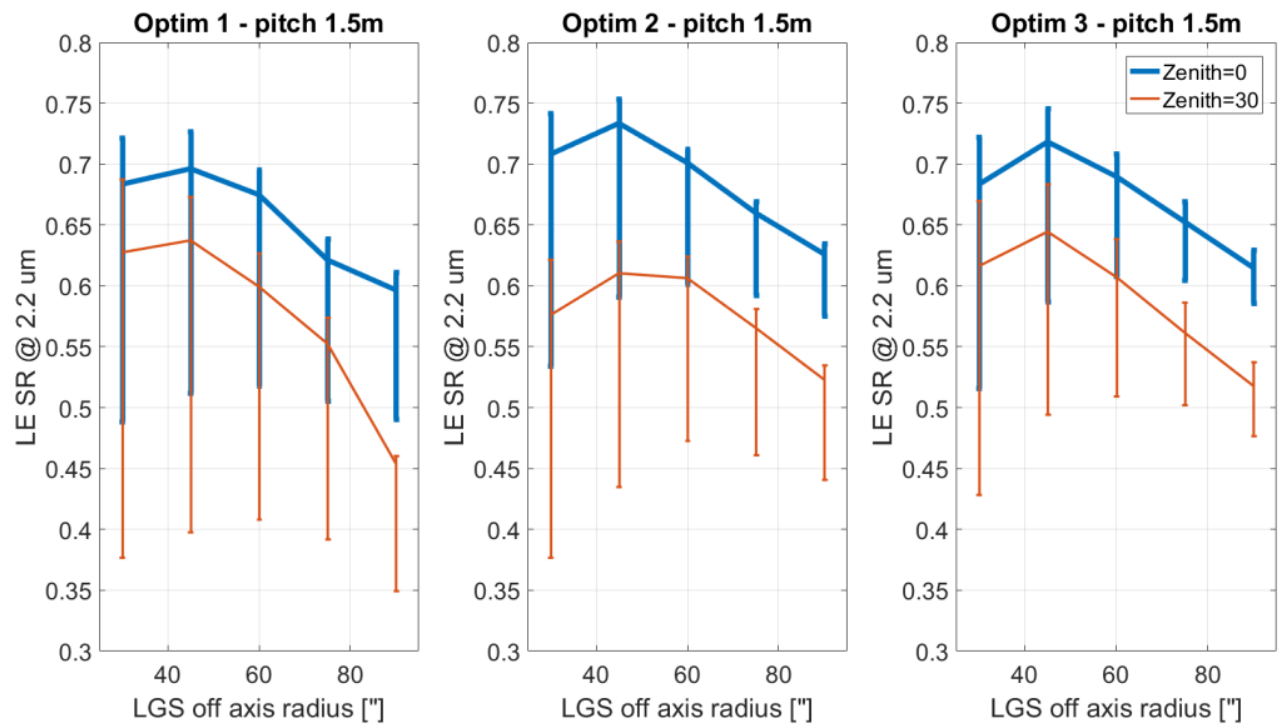

Figure 11: Maximum, mean across MICADO FoV and mean across 2' FoV of SR vs. LGS radius when optimizing tomography reconstruction over [Left] FoV1 [Middle] FoV2 [Right] FoV3 with pitch=1.5 m for zenith=0 (blue) and zenith $=30$ (red)

\subsection{Narrowest LGS constellation and baseline proposal}

An " ultimate performance" configuration is explored to evaluate how close it could make sense to bring the LGSs together. The NGSs are placed as close as possible to the science field, i.e. 30" radius accounting for the shadow of the pick-off mirror. The tomography projection is optimized in the MICADO small field 20"x20" and zenith distances as large as 60 degrees are considered. It is known that the asterism should be made narrower at larger zenith distances. For example, in the case of an on axis LTAO optimization, the optimal LGS constellation would be around 20" radius at airmass 2 .

This is confirmed by the results presented in Figure 12, where the average, minimum and maximum SR in the 20x20" field are plotted against LGS radius and for different zenith distances, with different $\mathrm{r} 0$ scaling:

- Left: the $\mathrm{r} 0$ is not scaled with zenith distance to see the performance trend only related to LGS geometry.

- Right: the $\mathrm{r} 0$ is scaled with zenith distance to witness a realistic performance gain (but without full error budget) in order to perform the right trade-off. 

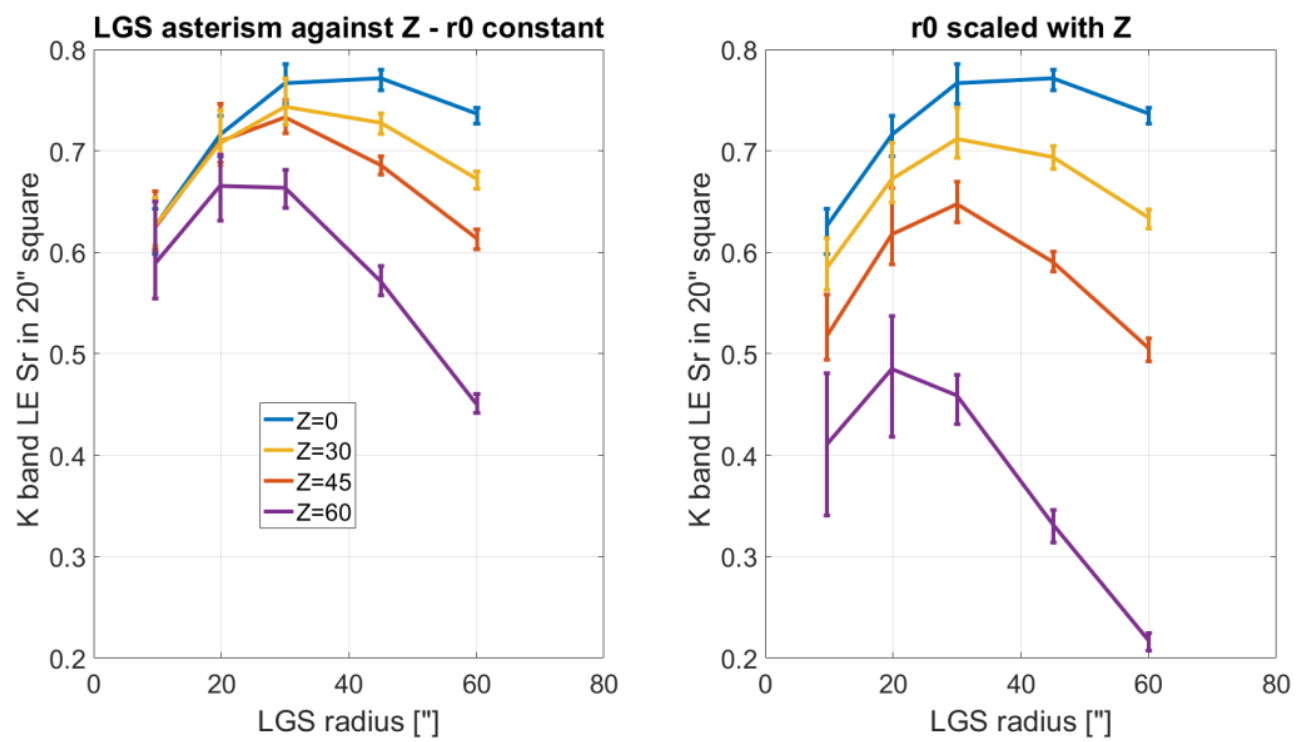

Figure 12: MAORY, r0=0.157m, Spec Cn2 profile, 2 DMs $1.5 \mathrm{~m}$ pitch, 3 NGS @ 30" radius - Average, maximum and minimum $K$ band SR in a 20"x20" square field, against LGS radius in " on sky, for 4 different zenith distances $(0,30,45$ and 60 degrees) [Left] r0 constant with Z [Right] r0 scaled with Z

For MICADO small field, an LGS radius of 30" is better in terms of ultimate scientific performance. 20" even improves further the performance on axis for $Z=60$, at the cost of uniformity. Nevertheless, the best compromise performance/uniformity corresponds to a fixed asterism @ 30" radius.

Based on these results, two baselines are possible for MAORY PDR:

1- Offer a wide field mode (LGSs@ @ 45" radius) and a narrow field mode (LGS@ 30" radius), respectively associated to MICADO large and small field. This would maximize the performance in the scientific field for both modes. The hexamid pick-off option would be required in this case.

2- Offer only a fixed 45" radius asterism based on the following considerations:

a. The ultimate small field performance would remain good for zenith distances smaller than 45 degrees. It can be considered that the high-performance mode is not suitable for large zenith distances.

b. The off-axis performance and hence the sky coverage would be better than what can be obtained with narrower LGS asterisms. Reminder: the results above have been generated with 3 NGSs @ 30" radius which is the best possible case in terms of quality of the corrected HO WF seen by the NGS WFSs.

c. The pick-off mirror design seems less risky and easier to align than the hexamid option, which would be required for LGS radii smaller than 45 ".

d. Having a fixed configuration would be in favor of robustness: One static, well aligned configuration avoid duplicating calibrations and associated maps (interaction matrix, NCPAs, etc.)

Finally, option 2 i.e. a fixed constellation at $45^{\prime \prime}$ radius is selected as MAORY's baseline for the sake of complexity minimization, hence higher robustness.

\section{CONCLUSION}

The MAORY design trade-off study has led to baseline the following items:

Number of post-focal DMs: provided that the error budget is kept under control, the specified performance can be achieved with one post-focal DM, however if budget allows, two post-focal DMs are desirable to enhance:

- Performance in the technical field to improve NGS sky coverage and robustness (ease acquisition)

- Scientific performance at all wavelength in presence of strong anisoplanatism

- Performance in the blue for NGS sky coverage and MICADO performance at lower wavelength

- Robustness to $\mathrm{Cn} 2$ profile variation and zenith distance 
Post-focal DMs pitch: $\sim 1.5 \mathrm{~m}$ projected onto the sky. Such pitch seems feasible and would be better than a $2 \mathrm{~m}$ pitch for worse seeing and with two post-focal DMs

Post-focal DMs altitude: $4-8$ and $16 \mathrm{~km}$ to be robust to larger airmass and accommodate two post-focal DMs in case of future upgrade

LGS asterism angle: fixed constellation at 45 " radius with optimization of tomography projection depending on FoV of interest

- E-ELT phase 1/phase 2:

- M1 doughnut would mostly impact sky coverage and PSF shape but has a moderate effect on the delivered Strehl ratio

- Six LGSs are highly desirable to reduce the tomographic error, particularly if the central obstruction of M1 is large

Before MAORY's PDR, we will concentrate the end-to-end simulation effort on the LGS WFS, in order to freeze a baseline design and address the associated risks such as the mitigation of spot truncation and NCPA management.

\section{REFERENCES}

[1] Diolaiti, E., "MAORY: A Multi-conjugate Adaptive Optics RelaY for the E-ELT", The Messenger 140, (2010).

[2] Diolaiti, E., Arcidiacono, C., Bregoli, G., Butler, R. C., Lombini, M., Schreiber, L., Baru olo, A., Basden, A., Bellazzini, M., Cascone, E., Ciliegi, P., Cortecchia, F., Cosentino, G., De Caprio, V., De Rosa, A., Dipper, N., Esposito, S., Foppiani, I., Giro, E., Morgante, G., Myers, R., Patru, F., Ragazzoni, R., Riccardi, A., Riva, M., Zerbi, F. M., Casali, M., Delabre, B., Hubin, N., Kerber, F., Le Louarn, M., Marchetti, E., Ramsay, S., Stroebele, S., and Vernet, E., "Preparing for the phase B of the E-ELT MCAO module project" in [Adaptive Optics Systems IV], Proc. SPIE 9148, 91480Y (2014).

[3] Diolaiti, E., Ciliegi, P., Abicca, R., Agapito, G., Arcidiacono, C., Baruffolo, A., Bellazzini, M., Biliotti, V., Bonaglia, M., Bregoli, G., Briguglio, R., Brissaud, O., Busoni, L., Carbonaro, L., Carlotti, A., Cascone, E., Correia, J.-J., Cortecchia, F., Cosentino, G., De Caprio, V., de Pascale, M., De Rosa, A., Del Vecchio, C., Delboulbe, A., Di Rico, G., Esposito, S., Fantinel, D., Feautrier, P., Felini, C., Ferruzzi, D., Fini, L., Fiorentino, G., Foppiani, I., Ghigo, M., Giordano, C., Giro, E., Gluck, L., H enault, F., Jocou, L., Kerber, F., La Penna, P., Lafrasse, S., Lauria, M., le Coarer, E., Le Louarn, M., Lombini, M., Magnard, Y., Maiorano, E., Mannucci, F., Mapelli, M., Marchetti, E., Maurel, D., Michaud, L., Morgante, G., Moulin, T., Oberti, S., Pareschi, G., Patti, M., Puglisi, A., Rabou, P., Ragazzoni, R., Ramsay, S., Riccardi, A., Ricciardi, S., Riva, M., Rochat, S., Roussel, F., Roux, A., Salasnich, B., Saracco, P., Schreiber, L., Spavone, M., Stadler, E., Sztefek, M.-H., Ventura, N., V erinaud, C., Xompero, M., Fontana, A., and Zerbi, F. M., "MAORY: adaptive optics module for the E-ELT" in [Adaptive Optics Systems V], Proc. SPIE 9909, 99092D (July 2016).

[4] Le Louarn, M. et al, "Simulations of Adaptive Optics Systems for the E-ELT", Proc SPIE 8447, 2012

[5] Tallon, M. et al, "Fractal iterative method for fast atmospheric tomography on extremely large telescopes", Proc SPIE 7736, 2010

[6] Arcidiacono, C., Schreiber, L., Bregoli, G., Diolaiti, E., Foppiani, I., Cosentino, G., Lombini, M., Butler, R. C., and Ciliegi, P., "End to end numerical simulations of the MAORY multiconjugate adaptive optics system" in [Adaptive Optics Systems IV], Proc. SPIE 9148, 91486F (2014).

[7] [Arcidiacono, C., Schreiber, L., Bregoli, G., Diolaiti, E., Foppiani, I., Agapito, G., Puglisi, A., Xompero, M., Oberti, S., Cosentino, G., Lombini, M., Butler, R. C., Ciliegi, P., Cortecchia, F., Patti, M., Esposito, S., and Feautrier, P., "The numerical simulation tool for the MAORY multiconjugate adaptive optics system" in [Adaptive Optics Systems V], Proc. SPIE 9909, 99097B (July 2016). 